\title{
ATITUDES DOS PROFESSORES UNIVERSITÁRIOS DOS CURSOS DE EDUCAÇÃO FÍSICA FACE À INCLUSÃO: UM ESTUDO EXPLO- RATÓRIO DE VALIDAÇÃO DA VERSÃO BRASILEIRA DO PEATID-III ${ }^{1}$
}

\author{
Soraya Dayanna Guimarães Santos \\ Universidade Federal de Alagoas, Maceió, Alagoas, Brasil \\ Neiza de Lourdes Frederico Fumes \\ Universidade Federal de Alagoas, Maceió, Alagoas, Brasil \\ José Pedro Ferreira \\ Universidade de Coimbra, Coimbra, Portugal
}

\begin{abstract}
Resumo
O estudo pretende adaptar e validar uma versão para o português do Brasil do Physical Educators' Attitudes toward Teaching Individuals with Disabilities-III (PEATID-III) e avaliar suas propriedades psicométricas. Participaram 76 professores de cursos de graduação em Educação Física de seis instituições de Educação Superior brasileira. As propriedades psicométricas foram analisadas com base no $r$ produto-momento de Pearson (teste-reteste), nos coeficientes de Alpha de Cronbach e na análise fatorial exploratória (com rotação Varimax). Os resultados revelaram uma estrutura global com dois fatores, que explicam 55,28\% da variância total. Concluiu-se que a validação da versão pode ser utilizada em docentes universitários dos cursos de Educação Física do Brasil.
\end{abstract}

Palavras-chave: Atitudes. Educação Física. Educação Superior. Educação Especial.

\section{Introdução}

As Instituições de Ensino Superior (IES) devem assegurar o pleno acesso aos estudantes com deficiência, em todas as atividades acadêmicas, considerando a Constituição Federal/1988, art. 205; o Aviso Circular no 277/MEC/GM/1996; o Decreto no 3.298/1999; o Decreto $n^{\circ} 3.956 / 2001$; a Lei $n^{\circ} 10.436 / 2002$; a Portaria $n^{\circ} 3.284 / 2003$; o Decreto $n^{\circ}$ 5.296/2004; o Decreto $\mathrm{n}^{\mathrm{o}}$ 5.626/2005; o Decreto $\mathrm{n}^{\mathrm{o}}$ 6.949/2009; o Decreto $\mathrm{n}^{\mathrm{o}} 7.234 / 2010$; o Decreto $\mathrm{n}^{\circ}$ 7.611/2011; e, a Lei n. ${ }^{\circ}$ 13.146/2015. No entanto, segundo Moreira (2004), estes documentos legais, embora muito importantes e necessários para uma educação inclusiva nas IES brasileiras, não garantem, por si só, a efetivação da inclusão propriamente dita (BRASIL, 1988; BRASIL, 1996; BRASIL, 1999; BRASIL, 2001; BRASIL, 2002; BRASIL, 2003; BRASIL, 2004; BRASIL, 2005; BRASIL, 2009; BRASIL, 2010; BRASIL, 2011; BRASIL, 2015).

Uma educação que prime pela inclusão deve ter, necessariamente: i) investimentos em materiais pedagógicos, em uma base legal específica que assegure a formação pedagógica do

\footnotetext{
${ }^{1} \mathrm{O}$ presente trabalho contou com apoio financeiro da Coordenação de Aperfeiçoamento de Pessoal de Nível Superior (CAPES), na forma de bolsa de doutorado.
} 
professor universitário; ii) investimento em infraestrutura adequada para ingresso, permanência e sucesso na aprendizagem, e iii) atenção a qualquer forma de discriminação.

É também necessário reconhecer que uma inclusão com sucesso depende largamente das atitudes dos professores para com o ensino de estudantes com deficiência (CAMPOS; FERREIRA; BLOCK, 2014), tendo como base o respeito à diferença. As atitudes dos professores assumem um papel importante e decisivo no processo de aprendizagem dos alunos, podendo se constituir em um auxílio ou um obstáculo à inclusão e, portanto, sendo um fator decisivo para a efetiva implementação deste processo (CARVALHO, 2011).

Os professores com atitudes positivas são mais propensos a ter intenção mais forte para a prática de inclusão (AJZEN; DRIVER, 1992). Geralmente, respeitam mais alunos com deficiência em suas aulas e, consequentemente, demonstram comportamento mais inclusivo (BARTOŇOVÁ; KUDLACEK; BRESSAN, 2007; MARTIN; KUDLACEK, 2010). Por outro lado, atitudes desfavoráveis dos professores podem afetar negativamente a aprendizagem de alunos com deficiência e, consequentemente, limitar o seu desenvolvimento global (PALLA; MAUERBERG-DECASTRO, 2004).

O número crescente de estudos sobre as atitudes dos professores face à inclusão de alunos com deficiência nas aulas de Educação Física realizados na última década enfatizou a importância das atitudes positivas como fator determinante para o sucesso da inclusão (BLOCK; OBRUSNIKOVA, 2007; ELLIOTT, 2008; COMBS; ELLIOTT; WHIPPLE, 2010; JERLINDER; DANERMARK; GILL, 2010; DOULKERIDOU et al., 2011; MAUERBERGDECASTRO et al., 2013). Todavia, outras variáveis associadas ao professor - tais como a idade, o sexo, a competência percebida, a perceção diante da experiência no ensino de alunos com deficiência, o percurso de formação acadêmica em Atividade Física Adaptada/ Educação Especial e o tipo de deficiência do estudante com quem o professor trabalha - são igualmente tidas como determinantes para o processo de inclusão de alunos com deficiência nas aulas de Educação Física (KOWALSKI; RIZZO, 1996; PAPADOPOULOU et al., 2004; MEEGAN; MACPHAIL, 2006; PETKOVA; KUDLÁČEK; NIKOLOVA, 2012; ÖZER et al., 2013).

A formação acadêmica relacionada às temáticas da Educação Inclusiva destaca-se dentre as variáveis que mais influenciam positivamente a atitude dos professores de Educação Física (FOLSOM-MEEK; RIZZO, 2002; AGUIAR; DUARTE, 2005; GUTIERRES FILHO et al., 2011). Entretanto, no contexto educacional brasileiro, pouquíssimos são os professores que tiveram uma formação específica nesta área durante a sua trajetória formativa, acarretando, com isso, o sentimento de insegurança e dificuldade em lidar com a diversidade (CASTANHO; FREITAS, 2006). De acordo com Duarte et al. (2013), a formação dos professores de Educação Física foi evidentemente técnica, competitiva e pouco pedagógica, além de não contar em seus currículos com conteúdos relativos à inclusão ou à pessoa com deficiência. Esta realidade é particularmente marcante em professores formados até o final da década de 1980 nas IES brasileiras.

É importante ressaltar que a formação dos professores não se encerra com o término da graduação, devendo se estender ao longo da vida profissional. Não obstante, Rizzo e Kirkendall (1995) advertem que a formação de professores de Educação Física não pode ser restrita apenas a uma disciplina, mas, sim, deve-se procurar dotar o currículo com infusões de conhecimentos e experiências ao longo de toda a trajetória acadêmica. É ainda consensual que a formação docente destes professores deve ter como foco o desenvolvimento de intenções, de crenças e de atitudes positivas em direção à inclusão de alunos com deficiência.

Para avaliar a atitude dos professores, é crucial o uso de instrumentos confiáveis e válidos. O Physical Educators' Attitude toward Teaching Individuals with Disabilities-III (PEATID III) (RIZZO, 1993) é um dos questionários mais utilizados em todo mundo para avaliar as atitudes dos professores sobre a inclusão de estudantes com deficiência nas aulas de Educação Física, tendo sido desenvolvido ao longo de diferentes etapas. A escala original Physi- 
cal Educators' Attitude toward Teaching the Handicapped (PEATH) foi desenvolvida em 1984, com um coeficiente de Alpha de Cronbach de 0,85. Entre 1986 e 1988, Rizzo realizou a validação do constructo aplicando a escala a uma amostra de 194 professores de Educação Física, tendo resultado em mudanças na nomenclatura e na terminologia dos tipos de deficiência da escala original, assim como no número de itens, que passou de 20 para 12, resultando em uma nova versão: o PEATH II (RIZZO, 1986, 1988).

Numa terceira fase, o PEATH II foi modificado e deu origem ao Physical Educators' Attitude toward Teaching Individuals with Disabilities-III (PEATID III) (RIZZO, 1993). Esta última revisão foi necessária para refletir uma terminologia mais atual e com um teor menos segregacionista. Folsom-Meek e Rizzo (2002) relataram a validade do constructo do PEATID-III, que foi obtida com a análise de componentes principais. A fidedignidade foi estimada por meio do valor do coeficiente de Alpha de Cronbach, tendo-se verificado $\alpha=0,88$ para a escala total.

O PEATID III mede três dimensões: a) os resultados face ao ensino de alunos com deficiência; b) os efeitos na aprendizagem dos alunos com deficiência, e c) a necessidade de mais formação acadêmica para ensinar os alunos com deficiência.

Com o uso generalizado da pesquisa, a obtenção de validade de constructo é essencial e fundamental para o cumprimento dos critérios de qualidade. Apesar de alguns estudos terem demonstrado a confiabilidade do instrumento, nenhum o utilizou anteriormente em professores do curso de Educação Física da Educação Superior. Assim, o presente estudo teve com principal objetivo adaptar e validar uma versão para o português do Brasil do Physical Educators' Attitudes toward Teaching Individuals with Disabilities-III (PEATID-III) e avaliar suas propriedades psicométricas quando utilizado com professores que lecionam nos cursos superiores de graduação em Educação Física.

\section{Método}

\section{Amostra}

Os participantes foram 76 professores de cursos de graduação (Licenciatura e Bacharelado) em Educação Física, com idades entre 24 e 66 anos, sendo 57,9\% (n= 44) do sexo masculino ( $M=41,75$ e $D P=10,42$ anos) e 42,1\% ( $n=32)$ do sexo feminino $(M=43,59$ e $\mathrm{DP}=10,19$ anos), pertencentes a seis IES do estado de Alagoas/Brasil, as quais uma era pública (com campi na capital e no interior) e cinco eram privadas (três localizadas na capital e duas no interior de Alagoas).

No que se refere à formação acadêmica dos docentes, constatamos que 44,7\% (n=34) dos inquiridos apresentavam o grau de mestre, 39,5\% ( $\mathrm{n}=30)$ haviam cursado especialização, $14,5 \%(\mathrm{n}=11)$ tinham o grau de doutor e $1,3 \%(\mathrm{n}=1)$ era pós-doutorado. No que se refere à formação em Atividade Física Adaptada (AFA)/Educação Especial (EE), 52,6 \% (n=40) dos professores de Educação Física afirmaram ter algum tipo de formação em AFA/EE obtido ao longo do seu percurso acadêmico. Em contraponto, 61,8\% dos docentes afirmaram nunca terem tido qualquer experiência de docência com estudantes com deficiência em suas aulas de Educação Física $(\mathrm{n}=47)$.

Foi possível verificar que 32,9\% $(\mathrm{n}=25)$ dos inquiridos apresentavam um tempo de serviço docente entre 6 meses e 5 anos, $21 \%(n=16)$ apresentavam um tempo de serviço entre 6 e 10 anos $(n=16)$, enquanto $17,1 \%(n=13)$ apresentavam um tempo de serviço entre 11 e 15 anos. Por último, 23,7\% $(\mathrm{n}=18)$ dos inqueridos apresentavam um tempo de serviço superior a 16 anos. Não responderam à questão formulada 5,3\% dos participantes $(\mathrm{n}=4)$.

O estudo foi submetido e aprovado pelo Comitê de Ética da Universidade Federal de Alagoas, Brasil (protocolo n ${ }^{\circ}$ 439.400). 


\section{Instrumento}

O instrumento de medida utilizado foi o PEATID III - Physical Educators' Attitude toward Teaching Individuals with Disabilities-III (FOLSOM-MEEK; RIZZO, 2002), adaptado para o português do Brasil, a partir da versão portuguesa adaptada por Campos, Ferreira e Block (2014).

O PEATID III apresenta diferentes seções. A primeira é composta 12 perguntas e exige que os professores expressem as suas atitudes face à ministração de aulas de Educação Física para estudantes com deficiência e exprimam os seus níveis de concordância ou discordância em relação a cinco deficiências: física, visual, auditiva, intelectual e múltipla. A resposta de cada um dos itens é realizada por meio de uma escala Likert que varia de 1 a 5 (1 discordo completamente; 2 - discordo; 3 - nem discordo nem concordo; 4 - concordo; 5 concordo completamente). A soma da pontuação de todas as respostas produzirá um resultado global que varia entre 12-60 pontos. O valor mais elevado indica uma atitude mais positiva diante do ensino de estudantes com deficiência. Há que se destacar a necessidade de recodificar os itens 5, 6, 7, 8, 9, 10, uma vez que estão formulados na negativa.

A segunda seção é composta de 14 perguntas (abertas e fechadas) e avalia os atributos (demográfico e descritivo) dos participantes, como sexo, idade, anos de experiência, experiência anterior no ensino de estudantes com deficiência, formação acadêmica em Educação Física Adaptada e Educação Especial, competência percebida e qualidade da experiência.

\section{Procedimentos para a produção dos dados}

Foram contactadas as coordenações dos cursos de Educação Física das diferentes IES, tendo sido apresentados os objetivos da pesquisa, prestados esclarecimentos sobre o desenho metodológico e sobre os riscos e benefícios da participação, assim como a confirmação do anonimato e da confidencialidade dos dados, conforme orientação da Resolução no 466/2012 sobre pesquisas com seres humanos, presente no Termo de Consentimento Livre Esclarecido (TCLE). Após obtido o consentimento de todas as coordenações, entramos em contato com os professores, presencialmente e via e-mail, com o objetivo de lhes apresentar o estudo, esclarecer eventuais dúvidas e solicitar a participação. Foram administrados os questionários a todos os docentes que aceitaram participar da pesquisa. O preenchimento foi realizado individualmente, tendo sido assegurado todo apoio e esclarecimento aos inquiridos durante o preenchimento.

No que se refere à adaptação do PEATID III para o português do Brasil, foram seguidas as etapas de validação transcultural descritas por Vallerand (1989) conducentes à preparação de uma versão experimental do instrumento. Esse processo envolveu adaptações linguísticas e culturais realizadas por um júri de três peritos, a partir da versão portuguesa do PEATID-III, uma vez que, no Brasil, o português falado e o escrito apresentam alterações semânticas, fonéticas e gramaticais substanciais que justificam tais modificações. Foram igualmente feitas adaptações em alguns itens, de modo a adequá-los ao contexto dos professores dos cursos de Educação Física da Educação Superior, mantendo a preocupação de não alterar seu significado. Este processo contou ainda com a realização de um estudo piloto $(n=6)$ para aferição de eventuais dificuldades na compreensão do questionário e de familiarização com as escalas de cotação.

\section{Procedimentos para a análise estatística dos dados}


A normalidade das variáveis em estudo foi analisada com a utilização do teste de Kolmogorov-Smirnov, com a correção de significância de Lilliefors e por meio da inspeção visual das curvas de normalidade dos gráficos. Em seguida, foi realizada uma análise fatorial exploratória (AFE) com rotação Varimax, com o objetivo de aferir a estrutura fatorial do PEATID-III e determinar a consistência interna das diferentes dimensões do instrumento. Para tal, foi utilizado como critério para a retenção de um item em um fator a apresentação de um valor de saturação igual ou superior a 0,30 nesse mesmo fator, correspondente à partilha entre eles de, pelo menos, $9 \%$ da variância total explicada (COHEN, 1992). A utilização desta técnica estatística foi possível devido ao fato de a dimensão da amostra utilizada cumprir um rácio mínimo de 5:1 (número de indivíduos para cada item do questionário), considerado adequado para a realização de uma análise fatorial exploratória (PASQUALI, 2006).

Foram ainda calculados os valores de média e desvio-padrão (estatística descritiva) de cada uma das dimensões obtidas para o PEATID III, assim como foi calculado o valor do coeficiente de Alpha de Cronbach para aferir a consistência interna de cada uma das dimensões do PEATID-III, e os valores de correlação item-total e de Alpha de Cronbach, se o item for apagado do fator. Estes procedimentos foram utilizados de modo a melhor compreender o peso relativo de cada um dos itens que integram as diferentes dimensões do instrumento em análise.

A fidedignidade teste-reteste foi calculada a partir dos valores do coeficiente de correlação $r$ produto-momento de Pearson, com um intervalo de aplicação de uma semana entre as duas aplicações do instrumento, e a sua estabilidade temporal foi testada com base nos valores do coeficiente de correlação intraclasse (ICC).

No que se refere à estatística inferencial, foram utilizados o teste T de Student para comparar as atitudes dos professores em estudo em função do sexo e a análise da variância ANOVA para comparar as atitudes dos professores em função dos anos de experiência docente. O tratamento estatístico dos dados foi realizado utilizando o software informático apropriado SPSS 21.0 ( ) for Windows. Todas as análises foram realizadas para uma probabilidade de erro associada de $5 \%$.

\section{Resultados}

Este estudo teve como objetivo adaptar e validar uma versão para o português do Brasil do Physical Educators' Attitudes Toward Teaching Individuals with DisabilitiesIII (PEATID-III) e avaliar as principais propriedades psicométricas.

\section{Dimensionalidade}

A dimensionalidade do PEATID-III foi analisada com base nos resultados da análise fatorial exploratória (AFE) com rotação Varimax, cujos pesos por fator ou dimensão são apresentados na Tabela 1. Os itens entre colchetes correspondem a itens que apresentaram pesos cruzados (cross-loadings).

Tabela 1 - Peso dos fatores do PEATID-III obtidos por meio da AFE, em professores dos cursos de graduação em Educação Física do estado de Alagoas $(\mathrm{N}=76)$

\begin{tabular}{lcccc}
\hline \multicolumn{1}{c}{ Subescalas } & Itens & F1 & F2 & F3 \\
\hline Efeitos na aprendiza- & 1 &, 83 & & \\
gem & 2 &, 77 & & \\
& 3 &, 50 & & \\
\hline & 4 &, 75 & & în
\end{tabular}




\begin{tabular}{lcccc}
\hline Resultados do ensino & 5 & &, 60 & \\
& 6 & &, 63 & \\
& 7 & &, 80 & \\
& 8 & & {$[, 59]$} & \\
& 9 & &, 68 &,- 66 \\
Preparação acadêmica & 10 & & {$[-, 45]$} &, 68 \\
& 11 & & & {$[, 57]$} \\
Valor próprio & 12 & 2,65 & 2,60 & 1,39 \\
\% variância & & 22,05 & 21,70 & 11,54 \\
\% var. acumulada & & 22,05 & 43,74 & 55,28 \\
\hline
\end{tabular}

Fonte: Elaborado pelos autores, 2016.

Ao contrário das três dimensões propostas por Folsom-Meek e Rizzo (2002) para a versão original do PEATID III, apresentadas na Tabela 2 e confirmadas para a versão de português de Portugal por Campos, Ferreira e Block (2014), a presente solução fatorial aponta para a existência de dois fatores que explicam 55,28\% da variância total. A maioria dos itens foi corretamente colocada nos respectivos fatores, sendo o número de pesos cruzados (crossloadings) relativamente baixo. $\mathrm{O}$ fator 1 , efeitos da aprendizagem, apresentou os 4 itens incluídos nos seus respectivos locais, replicando o fator original. $\mathrm{O}$ fator 2 , resultados da educação, apresenta 5 em 6 itens incluídos nos seus respectivos locais, embora os itens 8 e 10 apresentem peso cruzados (cross-loadings) com o fator 3. Por último, o fator 3, preparação acadêmica, originalmente constituído por 2 itens, apresentou pesos cruzados (cross-loadings) com o fator 2 nos itens 8 e 12 . Na sequência destes pesos cruzados, a sua estrutura final ficou reduzida a apenas 1 item, não podendo, portanto, ser considerada como um fator (NUNNALLY, 1978).

A Tabela 2 apresenta os valores de estatística descritiva (média e desvio-padrão) das atitudes dos professores dos cursos de Educação Física do de Alagoas/Brasil face ao ensino de estudantes com deficiência, tendo por base a estrutura fatorial do PEATID-III encontrada. São igualmente apresentados, em termos comparativos, os resultados do estudo de Campos, Ferreira e Block (2014), realizado com a versão portuguesa do mesmo instrumento, em professores portugueses do $3^{\circ}$ ciclo e do ensino secundário ${ }^{2}$ com idades compreendidas entre os $21 \mathrm{e}$ os 58 anos.

Tabela 2 - Valores de média e de desvio-padrão das diferentes dimensões do PEATID III nas versões de português do Brasil e de português de Portugal

\begin{tabular}{lcccc}
\hline Dimensões & \multicolumn{2}{c}{ Presente estudo } & \multicolumn{2}{c}{ Campos, Ferreira e Block (2014) } \\
& \multicolumn{2}{c}{$\mathrm{N}=76$} & $\mathrm{~N}=413$ \\
& $\mathrm{M}$ & $\mathrm{DP}$ & $\mathrm{M}$ & $\mathrm{DP}$ \\
\hline Efeitos na aprendizagem & 13,76 & 3,03 & 13,51 & 3,56 \\
\hline
\end{tabular}

\footnotetext{
${ }^{2}$ O Ensino Básico em Portugal compreende três ciclos: $1^{\circ}$ Ciclo: $1^{\circ}, 2^{\circ}, 3^{\circ}$ e $4^{\circ}$ Anos; $2^{\circ}$ Ciclo: $5^{\circ}$ e $6^{\circ}$ Anos; $3^{\circ}$ Ciclo: $7^{\circ}, 8^{\circ}$ e $9^{\circ}$ Anos. O Ensino Secundário é ministrado normalmente a jovens com idades compreendidas entre os 15 e os 18 anos e é constituído pelos $10^{\circ}, 11^{\circ}$ e $12^{\circ}$ Anos
} 


\begin{tabular}{lcccc}
\hline Resultados do ensino & 11,18 & 3,69 & 13,31 & 3,13 \\
Preparação acadêmica & - & - & 10,30 & 3,89 \\
\hline
\end{tabular}

Fonte: Elaborado pelos autores, 2016.

Os valores obtidos variam entre 13,76 $\pm 3,03$ (Efeitos da aprendizagem) e 11,18 $\pm 3,69$ (Resultados do ensino), sendo que são da mesma ordem de grandeza dos obtidos por Campos, Ferreira e Block (2014) e considerados ajustados, uma vez que nenhum dos itens ou dos fatores, isoladamente, apresentou um valor médio que se aproximasse dos valores extremos de 0 ou 5 (GUEDES; LEGNANI; LEGNANI, 2012).

\section{Consistência interna}

A consistência interna de cada uma das dimensões do PEATID III foi analisada utilizando os valores do coeficiente Alpha de Cronbach (ver Tabela 3).

Tabela 3 - Valores de correlação item-total, Alpha se o item for eliminado e Alpha de Cronbach para cada uma das dimensões da versão brasileira do PEATID III e respectivos itens ( $\mathrm{N}=76)$

\begin{tabular}{lcccc}
\hline \multicolumn{1}{c}{ Dimensões } & Itens & $\begin{array}{l}\text { Correlação } \\
\text { item-total }\end{array}$ & $\begin{array}{l}\text { Alpha de Cronbach } \\
\text { se item eliminado }\end{array}$ & $\begin{array}{c}\text { Alpha de Cro- } \\
\text { nbach }\end{array}$ \\
\hline Efeitos na aprendizagem & 1 & 0,601 & 0,63 & \\
& 2 & 0,539 & 0,67 & 0,73 \\
Resultados do ensino & 3 & 0,368 & 0,75 & \\
& 4 & 0,618 & 0,62 & \\
& 5 & 0,379 & 0,66 & \\
& 6 & 0,372 & 0,67 & \\
\hline & 7 & 0,625 & 0,54 & \\
& 9 & 0,491 & 0,61 & \\
& 10 & 0,351 & 0,67 & \\
\hline
\end{tabular}

Fonte: Elaborado pelos autores, 2016.

Os valores do coeficiente de Alpha de Cronbach obtidos para cada uma das dimensões encontradas foram tidos como aceitáveis (BRYMAN; CRAMER, 1993). De modo a aferir a contribuição de cada item para a consistência interna do fator, são igualmente apresentados os valores de Alpha quando o item é eliminado. Os resultados obtidos mostraram que, na maioria dos casos, os valores se mantinham muito próximos do valor de Alpha de Cronbach total.

\section{Fiabilidade teste-reteste}

Os resultados dos coeficientes de correlação intraclasse (CCI) e $r$ produto-momento de Pearson para cada uma das subescalas são apresentados na Tabela 4.

Tabela 4 - Coeficiente de correlação intraclasse e coeficientes de correlação de Pearson da versão brasileira do PEATID-III

\begin{tabular}{lcc}
\hline Subescalas do PEATID III & CCI & $\boldsymbol{R}$ \\
\hline Efeitos na aprendizagem & 0,73 & $0,88 * *$ \\
Resultados do ensino & 0,69 & $0,82 * *$ \\
\hline
\end{tabular}

** significativo para $\mathrm{p}<0,05$

Fonte: Elaborado pelos autores, 2016. 
Os valores dos coeficientes de correlação de Pearson (teste-reteste), calculados com base num intervalo de tempo entre aplicações de uma semana, variaram entre $r=0,88$ e $r=0,82$. No que se refere aos resultados obtidos para o CCI, estes confirmaram a existência de estabilidade temporal e discreta concordância para a dimensão resultados $(\mathrm{CCI}=0,69)$ e estabilidade temporal e elevada concordância para a dimensão efeitos $(\mathrm{CCI}=0,73)$.

\section{Validade de constructo}

O modelo original do PEATID-III, com três dimensões, foi reorganizado com base na presente amostra de 76 professores dos cursos de graduação (Licenciatura e Bacharelado) em Educação Física do estado de Alagoas/Brasil em duas dimensões: os efeitos de aprendizagem dos estudantes e os resultados da educação de estudantes com deficiência. O fator 3, preparação acadêmica, apresentou problemas de consistência interna, tendo perdido um item, pelo qual poderá ser objeto de análise mais detalhada em estudos futuros, envolvendo amostras de maior dimensão.

\section{Análise inferencial}

A Tabela 5 apresenta os valores de comparação das atitudes dos professores de Educação Física dos cursos de graduação (Licenciatura e Bacharelado) face à inclusão de estudantes com deficiência em função do sexo.

Tabela 5 - Comparação das atitudes dos professores de Educação Física face a inclusão em função do sexo

\begin{tabular}{lcccccc}
\hline \multicolumn{1}{c}{ Dimensões } & \multicolumn{2}{c}{$\begin{array}{c}\text { Masculino } \\
(\mathbf{n = 4 4 )}\end{array}$} & \multicolumn{2}{c}{$\begin{array}{c}\text { Feminino } \\
(\mathbf{n = 3 2})\end{array}$} \\
\hline & $\mathrm{M}$ & $\mathrm{Dp}$ & $\mathrm{M}$ & $\mathrm{Dp}$ & $p$ & $d$ Cohen \\
Resultados & 3,22 & 0,601 & 3,24 & 0,653 & 0,926 & 0,032 \\
Efeitos & 3,29 & 0,694 & 3,64 & 0,808 & $0,047^{* *}$ & 0,466 \\
\hline
\end{tabular}

** significativo para $\mathrm{p}<0,05$

Fonte: Elaborado pelos autores, 2016

Foram encontradas diferenças estatisticamente significativas entre homens e mulheres relativas às atitudes face à inclusão de alunos com deficiência nas aulas dos cursos graduação em Educação Física, no que se refere à dimensão efeitos na aprendizagem dos alunos $(\mathrm{p}<0,05)$. Foi igualmente encontrado um potencial efeito moderado do sexo $(d$ Cohen $=0,466)$ sobre as atitudes diante da inclusão de estudantes com deficiência nos cursos de Educação Física da Educação Superior. As docentes do sexo feminino apresentaram atitudes mais positivas face aos efeitos da inclusão ao nível da aprendizagem, quando comparadas aos seus colegas professores do sexo masculino. Referentes às atitudes dos professores em função dos anos de experiência docente, outra das variáveis em análise, não foram encontradas diferenças estatisticamente significativas entre os grupos em estudo.

\section{Discussão}

O presente trabalho pretendeu realizar um estudo exploratório de adaptação para português do Brasil do Physical Educators' Attitudes toward Teaching Individuals with Disabilities-III (FOLSOM-MEEK; RIZZO, 2002), validado para o português de Portugal por Campos, Ferreira e Block (2014), e aplicá-lo em uma amostra de professores de Educação Física dos cursos de graduação (Licenciatura e Bacharelado), no estado de Alagoas, Brasil. 
A investigação das atitudes dos professores universitários face à inclusão pode ser um diferencial para o processo de inclusão dos estudantes com deficiência em IES. Deste modo, torna-se fundamental a utilização de instrumentos válidos e confiáveis. No entanto, a validade não pode ser considerada como uma característica do instrumento per se e, sim, do instrumento aplicado a uma amostra, em que as características da população em estudo podem influenciar diretamente na estrutura de uma escala. Disto resulta a importância da presente pesquisa, confirmando a estrutura fatorial do instrumento numa amostra de docentes da Educação Superior, com características diferentes da anteriormente utilizada.

Pelos levantamentos realizados, este é o segundo estudo a utilizar o PEATID III com docentes de Educação Física da Educação Superior (SANTOS et al., [submetido]), uma vez que, até então, este só tinha sido utilizado com professores de Educação Física de escolas regulares da Educação Básica (DOULKERIDOU et al., 2011; FOURNIDOU; KUDLACEK; EVAGELLINOU, 2011) e com estudantes de Educação Física do último ano de graduação (PETKOVA; KUDLÁČEK; NIKOLOVA, 2012).

Os resultados obtidos apontaram para uma estrutura ligeiramente diferente da descrita no instrumento original (FOLSOM-MEEK; RIZZO, 2002), com uma redução do número de dimensões de três para duas.

Apesar da redução do número de fatores para dois, a dimensionalidade desta versão brasileira do PEATID-III consegue explicar, em conjunto, 55,28\% da variância total das atitudes dos professores face à inclusão de jovens com deficiência nas suas aulas de graduação em Educação Física na Educação Superior. A dimensão efeitos da aprendizagem apresentou uma boa consistência interna, uma boa confiabilidade e estabilidade temporal e uma discreta concordância, permitindo aferir a visão dos professores sobre os benefícios do trabalho conjunto com estudantes com e sem deficiência, favorecendo experiências mais motivadoras e ambientes de aprendizagens mais facilitadores e, ainda, avaliando o impacto no comportamento, tanto em sala de aula quanto na aprendizagem da aquisição de competências.

Por seu lado, a dimensão resultados da educação demonstrou uma aceitável consistência interna, uma boa confiabilidade e estabilidade temporal e uma elevada concordância, permitindo avaliar a opinião do professor sobre os resultados mais ou menos positivos da educação, associados à inclusão de jovens com deficiência nas aulas de Educação Física dos cursos de graduação no Educação Superior.

Os resultados do presente estudo são semelhantes aos obtidos por Campos, Ferreira e Block (2014), com uma amostra de docentes do ensino básico, os quais apontam igualmente para a existência de duas dimensões claramente definidas, os efeitos da aprendizagem e os resultados da educação, sendo que a dimensão preparação acadêmica apresentava, em semelhança ao presente estudo, problemas de consistência interna, com valores de Alpha de Cronbach inferiores ao mínimo aceitável. Por se tratar de um estudo com características pioneiras em termos de aplicação no contexto da Educação Superior, a comparação dos resultados obtidos com os de outros estudos constitui uma limitação que é importante assinalar.

Uma parte significativa dos estudos publicados na literatura sobre a temática das atitudes face à inclusão aponta para o fato de as docentes do sexo feminino apresentarem atitudes mais favoráveis à inclusão de jovens com deficiência nas aulas de Educação Física, quando comparadas com os seus colegas docentes do sexo masculino (ALOIA et al., 1980; DOWNS; WILLIAMS, 1994; FOLSOM-MEEK et al., 1999; CONATSER; BLOCK; LEPORE, 2000; HUTZLER; ZACH; GAFNI, 2005). No entanto, outros estudos apontam para a não existência de diferenças entre docentes do sexo masculino e docentes do sexo feminino (KOWALSKI; RIZZO, 1996; RIZZO; VISPOEL, 1991; RIZZO; KIRKENDALL, 1995), tornando esta alegada diferença entre sexos inconsistente e inconclusiva. Contudo, Kowalsi e Rizzo (1996) reconheceram que, apesar de não terem encontrado diferenças estatisticamente significativas no que se refere às atitudes dos docentes do sexo masculino e feminino face à inclusão de 
estudantes com deficiência nas aulas de Educação Física dos cursos de graduação na Educação Superior, as docentes do sexo feminino apresentavam atitudes mais favoráveis, isto é, valores médios mais elevados que apontavam no sentido da inclusão.

O presente estudo reforça o resultado mencionado anteriormente, confirmando a existência de diferenças significativas entre a perceção dos professores do sexo masculino e do sexo feminino diante da inclusão, em especial, no que se refere aos seus efeitos na aprendizagem dos alunos.

Salientamos que este resultado foi reforçado pela existência de um potencial efeito moderado do sexo sobre as atitudes quanto à inclusão de estudantes com deficiência nas aulas de Educação Física dos cursos superiores (Licenciatura e Bacharelado). As docentes do sexo feminino apresentavam atitudes mais positivas e valorizavam mais os efeitos da inclusão ao nível da aprendizagem.

O presente estudo apresenta algumas limitações, sendo a mais relevante o número reduzido de docentes do Educação Superior avaliados, o que impossibilita a realização de uma análise fatorial confirmatória, procedimento estatístico mais adequado para a validação do questionário visando a uma ampla utilização em professores brasileiros dos cursos de graduação (Licenciatura e Bacharelado) em Educação Física. Para além disso, parece ter ocorrido uma interpretação ambígua dos itens 8 e 12 do PEATD-III por parte dos professores brasileiros dos cursos de graduação em Educação Física, provavelmente baseada numa interpretação e numa compreensão diferentes dos conceitos a eles associados, o que sugere uma futura necessidade de revisão e de reformulação dos referidos itens, em especial, do item 8 , determinante para o funcionamento da dimensão relativa às atitudes no contexto da preparação acadêmica.

\title{
Conclusões
}

O presente estudo demonstrou que a versão em português do Brasil do PEATID-III tem propriedades psicométricas adequadas, com índices aceitáveis de consistência interna e de confiabilidade que permitem avaliar as atitudes dos professores dos cursos de graduação (Licenciatura e Bacharelado) em Educação Física do estado de Alagoas/Brasil, para o ensino de estudantes com deficiência na Educação Superior, nas dimensões efeitos da aprendizagem e resultados da educação. No entanto, é necessário ter atenção ao fato de que este estudo constitui um primeiro passo na validação transcultural deste instrumento de avaliação psicológica, sendo necessário, em estudos futuros, aferir com maior detalhe o comportamento dos itens da dimensão preparação acadêmica, bem como proceder a uma análise mais detalhada da estrutura fatorial do instrumento por meio da realização de uma análise fatorial confirmatória.

\section{Agradecimentos}

Agradecemos à Coordenação de Aperfeiçoamento de Pessoal de Nível Superior (CAPES) pelo fomento e pelo financiamento deste estudo.

\section{PHYSICAL EDUCATION UNIVERSITY TEACHER'S ATTITUDES TOWARD IN- CLUSION: AN EXPLORATORY STUDY FOR THE VALIDATION OF THE BRA- ZILIAN VERSION OF PEATID-III}

\author{
Abstract \\ Pensar a Prática, Goiânia, v. 21, n. 1, jan./mar. 2018
}


The study aims to adapt and validate a Brazilian Portuguese version of Physical Educators' Attitudes towards Teaching Individuals with Disabilities-III (PEATID-III) and to evaluate its psychometric properties. Participated 76 teachers of undergraduate courses in Physical Education of six Brazilian higher education institutions. The psychometric properties were analyzed based on Pearson's product-moment (test-retest), Cronbach's Alpha coefficients and exploratory factorial analysis (with Varimax rotation). The results revealed a global structure with two factors, which explain $55.28 \%$ of the total variance. It was concluded that the validation of the version can be used in university professors of Physical Education courses in Brazil.

Keywords: Attitudes. Physical Education. Higher Education. Special Education.

\section{ACTITUDES DE DOCENTES UNIVERSITARIOS DE LOS CURSOS DE EDUCA- CIÓN FÍSICA EN RELACION CON LA INCLUSIÓN: UN ESTUDIO EXPLORATO- RIO DE VALIDACIÓN DE LA VERSIÓN BRASILEÑA DEL PEATID-III}

\section{Resumen}

El estudio tuvO como objetivo adaptar y validar una versión para el portugués de Brasil del cuestionario Physical Educators' Attitudes towards Teaching Individuals with Disabilities-III (PEATID-III) y evaluar sus propiedades psicométricas. Participaron 76 profesores de los cursos de graduación en Educación Física de seis instituciones de educación superior brasileña. Las propiedades psicométricas fueron analizadas con base en el momento-producto de Pearson $\mathrm{r}$ (test-retest), el coeficiente alfa y el análisis factorial exploratorio (con rotación Varimax) de Cronbach. Los resultados revelaron una estructura general con dos factores que explican $55.28 \%$ de la varianza total. Se concluyó que la validación de la versión se puede utilizar en los profesores universitarios de cursos de Educación Física en Brasil.

Palabras clave: Actitudes. Educación y Entrenamiento Físico. Educación Superior. Educación Especial.

\section{Referências}

AGUIAR, J. S.; DUARTE, E. Educação inclusiva: um estudo na área da Educação Física. Revista Brasileira de Educação Especial, Marília, v. 11, n.2, p. 223-240, maio/ago., 2005.

AJZEN, I.; DRIVER B. L. Application of the theory of planned behavior to leisure choice. Journal of Leisure Research, v. 24, n.3, p. 207-224, fev./maio, 1992.

ALOIA, G. F. et al. Physical education teachers' initial perceptions of handicapped children. Mental retardation, v. 18, n. 2, p. 85-87, 1980.

BARTOŇOVÁ, R.; KUDLÁČEK, M.; BRESSAN, L. Attitudes of future physical educators toward teaching children with disabilities in physical education in the Republic of South Africa. Acta. Univ. Palacki. Olomuc. Gymn, v. 37, n. 4, p. 69-75, 2007.

BLOCK, M. E.; OBRUSNIKOVA, I. Inclusion in physical education: A review of the literature from 1995-2005. Adapted Physical Activity Quarterly, v. 24, n.2, p.103-124, 2007.

BRASIL. Lei n. ${ }^{\circ} 13.146$ de 6 de julho de 2015. Institui a Lei Brasileira de Inclusão da Pessoa com Deficiência (Estatuto da Pessoa com Deficiência). Brasília, 2015. 
Constituicão da República Federativa do Brasil de 1988. Brasília, 1988. Disponível em:

〈http://www.planalto.gov.br/ccivil 03/constituicao/constituicao.htm>. Acesso em: 27 maio 2016.

. Aviso Circular N 277/MEC/GM/1996. A execução adequada de uma política educacional dirigida aos portadores de necessidades especiais possibilita que venham a alcançar níveis cada vez mais elevados do seu desenvolvimento acadêmico. Brasília, 1996. Disponível em:

〈http://portal.mec.gov.br/seesp/arquivos/pdf/aviso277.pdf >. Acesso em: 27 maio 2016.

Lei $\mathrm{n}^{\circ} 3.298$ de 20 de dezembro de 1999 . Que regulamenta a Lei $\mathrm{n}^{\circ} 7.853$, propõe equiparação de oportunidades as pessoas com deficiência. Brasília, 1999. Disponível em: 〈http://www.planalto.gov.br/ccivil_03/decreto/d3298.htm>. Acesso em: 27 maio 2016.

. Decreto $n^{\circ} 3956$ de 8 de outubro de 2001. Promulga a Convenção Interamericana para a Eliminação de Todas as Formas de Discriminação contra as Pessoas Portadoras de Deficiência. Brasília: 2001. Disponível em:

<http://www2.senado.leg.br/bdsf/bitstream/handle/id/43/Livro\%20\%20Acessibilidade.pdf?se q ence=5>. Acesso em: 27 maio 2016.

. Lei $n^{\circ} 10.436$ de 24 de abril de 2002. Dispõe sobre a Língua Brasileira de Sinais Libras e dá outras providências. Brasília: 2002. Disponível em: <http://www.planalto.gov.br/ccivil_03/leis/2002/110436.htm>. Acesso em: 27 maio 2016.

Portaria $\mathrm{n}^{\circ} 3.284$, de 7 de novembro de 2003. Dispõe sobre requisitos de acessibilidade de pessoas portadoras de deficiências, para instruir os processos de autorização e de reconhecimento de cursos, e de credenciamento de instituições. Brasília, 2003. Disponível em:

〈http://portal.mec.gov.br/arquivos/pdf/portaria3284.pdf $>$. Acesso em: 27 maio 2016.

. Decreto ${ }^{\circ} 5.296$ de 2 de dezembro de 2004. Normas gerais e critérios básicos para a promoção da acessibilidade das pessoas portadoras de deficiência ou com mobilidade reduzida. Brasília: 2004. Disponível em:

<http://www.planalto.gov.br/ccivil_03/_Ato2004-2006/2004/Decreto/D5296.htm>. Acesso em: 27 maio 2016.

. Decreto $\mathrm{n}^{\circ}$ 5.626, de 22 de dezembro de 2005. Dispõe sobre a Língua Brasileira de Sinais - Libras, e o art. 18 da Lei $\mathrm{n}^{0}$ 10.098, de 19 dezembro de 2000. Brasília, 2005. Disponível em:

<http://www.planalto.gov.br/ccivil 03/ ato2004-2006/2005/decreto/d5626.htm>. Acesso em: 27 maio 2016.

Decreto $\mathrm{n}^{\circ}$ 6.949, de 25 de agosto de 2009. Promulga a Convenção Internacional sobre os Direitos das Pessoas com Deficiência e seu Protocolo Facultativo, assinados em Nova York, em 30 de março de 2007. Brasília, 2009. Disponível em: 〈http://www.planalto.gov.br/ccivil_03/_ato2007-2010/2009/decreto/d6949.htm>. Acesso em: 27 maio 2016. 
Decreto $\mathrm{n}^{\circ} 7.234$, de 19 de julho de 2010. Dispõe sobre o Programa Nacional de Assistência Estudantil - PNAES. Brasília, 2010. Disponível em:

<http://www.planalto.gov.br/ccivil_03/_Ato2007-2010/2010/Decreto/D7234.htm>. Acesso em: 27 maio 2016.

Decreto $n^{\circ} 7.611$, de 17 de novembro de 2011. Dispõe sobre a educação especial, o atendimento educacional especializado e dá outras providências. Brasília, 2011. Disponível em: 〈http://www.planalto.gov.br/ccivil_03/_ato2011-2014/2011/decreto/d7611.htm>. Acesso em: 27 maio 2016.

Lei n. ${ }^{\circ} 13.146$ de 6 de julho de 2015. Institui a Lei Brasileira de Inclusão da Pessoa com Deficiência (Estatuto da Pessoa com Deficiência). Brasília, 2015. Disponível em: <http://www.planalto.gov.br/ccivil_03/_ato2015-2018/2015/lei/113146.htm>. Acesso em: 24 out. 2017.

BRYMAN, A.; CRAMER, D. Análise de dados para Ciências Sociais: a utilização do SPSS. Oeiras: Celta Editora, 1993.

CAMPOS, M. J.; FERREIRA, J. P.; BLOCK, M. E. Analyzing the structure, validity and reliability of the Physical Educators'Attitude Toward Teaching Individuals with Disabilities - III (PEATID-III). Annals of Research in Sport and Physical Activity, Coimbra, n. 5, p. 102114, 2014.

CARVALHO, M. S. F. As atitudes dos professores face à inclusão de alunos com deficiência - o contacto com a deficiência. 2011. 78 f. Dissertação (Mestrado em Ensino da Educação Física) - Curso de Mestrado em Ensino de Educação Física no Ensino Básico e Secundário, Universidade Lusófona de Humanidades e Tecnologias, Lisboa, 2011.

CASTANHO, D. M.; FREITAS, S. N. Inclusão e prática docente no ensino superior. Revista Educação Especial, Santa Maria, v. 27, n. 1, p. 93-99, 2006.

COHEN, J. A power primer. Psychological Bulletin, Berkeley, v. 112, p.155-159, 1992

COMBS, S.; ELLIOTT, S.; WHIPPLE, K. Elementary physical education teachers' attitudes towards the inclusion of children with special needs: a qualitative investigation. International Journal of Special Education, Vancouver, v. 25, n. 1, p. 114-125, 2010.

CONATSER, P.; BLOCK, M.; LEPORE, M. Aquatic instructors' attitudes toward teaching students with disabilities. Adapted Physical Activity Quarterly, v. 17, n. 2, p. 197-207, 2000.

DOULKERIDOU, A. et al. Attitudes of Greek Physical Education teachers towards inclusion of students with disabilities in physical education classes. International Journal of Special Education, v. 26, n. 1, p.1-11, 2011.

DOWNS, P.; WILLIAMS, T. Student attitudes toward integration of people with disabilities in activity settings: A European comparison. Adapted Physical Activity Quarterly, v. 11, p.32-32, 1994. 
DUARTE, E. R. et al. Estudo de caso sobre a inclusão de alunos com deficiência no Ensino Superior. Revista Brasileira de Educação Especial, v. 19, n. 2, p. 289-300, 2013.

ELLIOTT, S. The effect of teachers' attitude toward inclusion on the practice and success levels of children with and without disabilities in Physical education. International Journal of Special Education, v. 23, n. 3, p. 48-55, 2008.

FOLSOM-MEEK, S. L. et al. Effects of academic major, gender, and hands-on experience on attitudes of preservice professionals. Adapted Physical Activity Quarterly, v. 16, p.389402, 1999.

FOLSOM-MEEK, S. L.; RIZZO, T. L. Validating the Physical Educators' Attitude Toward Teaching Individuals With Disabilities III (PEATID III) Survey for Future Professionals. Adapted Physical Activity Quarterly, v. 19, n. 2, p. 141-154, 2002.

FOURNIDOU , I.; KUDLACEK , M.; EVAGELLINOU, C. Attitudes of in-service physical educators toward teaching children with physical disabilities in general physical education classes in Cyprus. European Journal of Adapted Physical Activity, v. 4, n. 1, 2011.

GUEDES, D. P.; LEGNANI, R. F. S.; LEGNANI, E. Propriedades psicométricas da versão brasileira do Exercise Motivations Inventory (EMI-2). Revista Motriz, Rio Claro, v. 18, n. 4, p. 667-677, out./dez., 2012.

GUTIERRES FILHO, P. J. B. et al. Concepções, opiniões e atitudes docentes associadas à inclusão da pessoa com deficiência na Educação Física: Uma revisão da produção científica brasileira. Liberabit, v.17, n.1, p. 19-30, mar., 2011.

HUTZLER, Y.; ZACH, S.; GAFNI, O. Physical education students' attitudes and self- efficacy towards the participation of children with special needs in regular classes. European Journal of Special Needs Education, v. 20, n. 3, p. 309-327, 2005.

JERLINDER, K.; DANERMARK, B.; GILL, P. Swedish primary- school teachers' attitudes to inclusion - the case of PE and pupils with physical disabilities. European Journal of Special Needs Education, v. 25, n. 1, p. 45-57, fev., 2010.

KOWALSKI, E. M.; RIZZO, T. L. Factors influencing preservice student attitudes toward individuals with disabilities. Adapted Physical Activity Quarterly, v. 13, p. 180-196, 1996.

MARTIN, K.; KUDLÁČEK, M. Attitudes of pre-service teachers in an Australian university towards inclusion of students with physical disabilities in general physical education programs. European Journal of Adapted Physical Activity, v.3, n.1, p. 30-48, 2010.

MAUERBERG-DECASTRO, E. M. et al. Attitudes about inclusion by educators and physical educators: Effects of participation in an inclusive adapted physical education program. Revista Motriz, Rio Claro, v. 19, n. 3, p. 649-661, 2013.

MEEGAN, S.; MACPHAIL, A. Irish physical educators' attitude toward teaching students with special educational needs. European Physical Education Review, v. 12, n. 1, p. 75-97, 2006. 
MOREIRA, L. C. Universidade e alunos com necessidades educacionais especiais: das ações institucionais às práticas pedagógicas. 2004. 233f. Tese (Doutorado) - Faculdade de Educação, Universidade de São Paulo, São Paulo, 2004.

NUNNALLY, J. C. Psychometric theory. New York: McGraw-Hill, 1978

ÖZER, D. et al. Physical education teachers' attitudes towards children with intellectual disability: the impact of time in service, gender, and previous acquaintance. Journal of Intellectual Disability Research, v. 57, n. 11, p. 1001-1013, nov., 2013.

PALLA, A. C.; MAUERBERG-DECASTRO, E. Atitudes de professores e estudantes de Educação Física em relação ao ensino de alunos com deficiência em ambientes inclusivos. Revista da Sobama, Rio Claro, v. 9, n. 1, p. 25-34, dez., 2004.

PAPADOPOULOU, D. et al. Attitudes of Greek physical education teachers toward inclusion of students with disabilities. International Journal of Special Education, Vancouver, v. 19, n. 2, p. 104-111, 2004.

PASQUALI, L. Análise fatorial para psicólogos. Brasília: LabPAM. 2006.

PETKOVA, A.; KUDLÁČEK, M.; NIKOLOVA, E. Attitudes of physical education students (last university year) and physical education teachers toward teaching children with physical disabilities in general physical education classes in Bulgaria. European Journal of Adapted Physical Activity, v. 5, n. 2, p. 82-98, 2012.

RIZZO, T.L. Physical educators' attitude toward teaching the handicapped II. San Bernardino, CA: California State University, 1986. [Unpublished survey].

RIZZO, T. L.; WRIGHT, R. G. Physical educators' attitudes toward teaching students with handicaps. Mental Retardation, v. 26, p. 307-309, 1988.

RIZZO, T. L. Physical educators' attitude toward teaching individuals with disabilitiesIII. San Bernardino, CA: California State University,1993. [Unpublished survey].

RIZZO, T. L.; VISPOEL, W. P. Physical educators' attributes and attitudes toward teaching students with handicaps. Adapted Physical Activity Quarterly, v. 8, p. 4-11, 1991.

RIZZO, T. L.; KIRKENDALL, D. Teaching Students with Mild Disabilities: What Affects Attitudes of Future Physical Educators? Adapted Physical Activity Quarterly, v. 12, p. 205216, jul., 1995.

SANTOS, S. D. G. et al. Higher Education Physical Education Teachers Attitudes and Perceived Competence toward Teaching Students with Disability. European Journal of Teacher Education. [Submitted].

VALLERAND, R. J. Vers une méthodologie de validation trans-culturelle de questionnaires psychologiques: implications pour la recherche en langue française. Canadian Psychology/Psychologie Canadienne, v. 30, n. 4, p. 662-680, out., 1989. 
Recebido em: 21/06/2016

Revisado em: 27/08/2017

Aprovado em: 27/11/2017

Endereço para correspondência:

soraya dayanna@hotmail.com

Soraya Dayana Guimarães Santos

Universidade Federal de Alagoas, Centro de Educação.

Campus A.C. Simões

Tabuleiro dos Martins

57072-970 - Maceio, AL - Brasil 